und Institutionen, Arbeitslosigkeit und wirtschaftliches Wachstum, Aus-, Ab-, oder Umbau des Wohlfahrtsstaates etc.)? Welche individuellen wie gesellschaftlichen Leistungen und Erfolge sind an diese Vermögensart gekoppelt? Welche negativen Effekte sind mit dieser Kapitalart verbunden?

Wie wird soziales Kapital aufgebaut und erhalten? Welche Individuen oder auch Gesellschaften zeichnen sich durch einen hohen Bestand an sozialem Kapital aus, welche durch einen Mangel und warum? Lassen sich Verbindungen $\mathrm{zu}$ anderen Forschungsrichtungen, z.B. Kooperationsforschung und Spieltheorie, fruchtbar nutzen?

Die Tagung zeigt sich offen für theoretische ebenso wie stärker empirisch fundierte Beiträge, wobei es sich um individualdaten- oder aggregatdatenbasierte Analysen oder auch z.B. komparative Fallstudien handeln kann.

Für nähere Informationen wenden Sie sich bitte an Andreas Diekmann, E-Mail: diekmann@soz.gess.ethz.ch, Markus Freitag, E-Mail: freitag@soz.gess.ethz.ch oder Ben Jann, E-Mail: jann@soz.gess.ethz.ch, Professur für Soziologie, ETH Zentrum SEW E 21, 8092 Zürich, Schweiz

"Menschen in der Illegalität. Lebensbedingungen von Papierlosen als Herausforderung für die Kommunen"

Kriminologische Studienwoche vom 27. Sept. bis 1. Okt. 2004 in der Universität Hamburg

Es wird geschätzt, dass derzeit bis zu 1 Million Menschen illegal in Deutschland leben. Diese „illegalen MigrantInnen" sind entweder ohne Aufenthaltserlaubnis nach Deutschland eingereist oder haben sie hier verloren - sie sind „Papierlose", die sich von allen anderen Zuwanderergruppen dadurch unterscheiden, dass ihr bloßer Aufenthalt bereits einen Rechtsbruch darstellt. Diese Menschen kommen weder in dem Koalitionsabkommen der regierenden Parteien vor, noch im Zuwanderungsgesetz - und bisher auch nicht in der Kriminologie. Gleichzeitig werden Jahr für Jahr mehr personelle und finanzielle Ressourcen zur Abwehr von illegaler Migration eingesetzt. Die Lebenssituation und die Lebensbedingungen dieser Menschen sind in der Öffentlichkeit weitgehend unbekannt. Auch die Rückwirkungen der Abwehr von MigrantInnen und der Suche nach
„Illegalen“ für Demokratie und Rechtsstaat bleiben öffentlich undiskutiert.

Mit der Studienwoche soll der Erfahrungsund Informationsaustausch derjenigen unterstützt werden, die mit dieser Thematik in Berührung kommen (der Bereiche Sozialarbeit, Polizei, Justiz, Medizin u.v.a.m.). Wie sehen sich die verschiedenen Professionen - von der Strafverfolgung über die Kirche bis zur Schule - damit konfrontiert? Welche Schwierigkeiten ergeben sich in ihrer Arbeit? Welche Handlungsmöglichkeiten haben PraktikerInnen und kommunalpolitisch Aktive? Dies sind nur der zentralen Fragen, denen in dieser Woche in einer interdisziplinären Runde nachgegangen werden wird.

Der Bewerbungsschluss ist der 24. Juni 2004, die Teilnahmegebühr beträgt $248 €$. Weitere Informationen erhalten Sie von: Bettina Paul, Universität Hamburg, Institut für Kriminologische Sozialforschung, Allende-Platz 1, D-20146 Hamburg, E-Mail: bettina.paul@uni-hamburg.de, Tel.: 040-42838-3322, Sekretariat: 040-428383329, Fax: 040-42838-2328 oder unter www.rrz.uni-hamburg.de/kriminol/welcome.htm.

\section{Aufbaustudium Kriminologie}

Beginn des nächsten Studiengangs im Sommersemester 2005

Im Sommersemester 2005 beginnt der nächste Durchgang des 4-semestrigen Aufbaustudiums Kriminologie mit einem neuen Curriculum.

Das Studium der Kriminologie wird zukünftig eine stärkere internationale Ausrichtung haben und vergleichende Perspektiven in den zwei Schwerpunkten Policing und Gewalt unter der Perspektive Governing Security vermitteln.

Die Voraussetzungen sind ein abgeschlossenes Studium in Soziologie, Psychologie, Pädagogik, Humanmedizin, Rechtswissenschaft oder verwandten Fächern, oder ein abgeschlossenes Fachhochschulstudium, wie z.B. Sozialpädagogik oder Verwaltung. Des Weiteren sind die überdurchschnittliche Befähigung zum wissenschaftlichen Arbeiten und Fremdsprachenkenntnisse, insbesondere im Englischen, Voraussetzung. Erwünscht ist eine vorherige wissenschaftliche Beschäftigung mit kriminologischen Problemfeldern.

Bewerbungen sind in der Zeit vom 15.12. 2004 bis 15.01.2005 (Ausschlussfrist) an das Aufbau- und Kontaktstudium Kriminologie der 